\title{
Prevalence and Correlates of Kaposi'S Sarcoma-Associated Herpesvirus and Herpes Simplex Virus Type 2 Infections Among Adults: Evidence from the NHANES III Data
}

\author{
Xin Zhang \\ Fudan University \\ Yiyun Xu \\ Fudan University \\ Yi Li \\ Fudan University \\ Huangbo Yuan \\ Fudan University \\ Zhenqiu Liu \\ Fudan University \\ Tiejun Zhang ( $\nabla$ tjzhang@shmu.edu.cn ) \\ Fudan University
}

\section{Research}

Keywords: KSHV, HSV-2, risk factor, NHANES

Posted Date: September 13th, 2021

DOI: https://doi.org/10.21203/rs.3.rs-868386/v1

License: (c) (i) This work is licensed under a Creative Commons Attribution 4.0 International License. Read Full License 


\section{Abstract}

Background Kaposi's sarcoma-associated herpes virus (KSHV) prevalence and risk factors exhibit considerable variations across populations in different geographic regions. Determinants and the transmission routes of KSHV infection are uncertain. We seek to identify the possible risk factors and the transmission routes of KSHV infection in non-endemic areas.

Methods We collected annual cases and seroprevalence of KSHV and herpes simplex virus type 2 (HSV-2) from the NHANES III sampled individuals from the US general population (1988-1994). We included 13179 and 10720 individuals with available remaining serum samples of KSHV and HSV-2. Logistic regression were employed to explore potential risk factors for the seropositivity.

Results The seroprevalence was $2.05 \%$ for KSHV infection and $31.03 \%$ for HSV2 infection among this population. All risk factors of sexual behaviors included were strongly associated with HSV-2 positive, however, only MSM had an approximately fivefold increased risk of KSHV infection (OR=4.71; 95\% Cl 1.61 11.30). Mexican Americans (2.51\%) and older (chi-sqaure trend $=-6.71, P<0.001)$ individuals had a higher risk of KSHV infection. After adjustment, individuals with higher level of education and economic status had lower KSHV infection.

Conclusions In non-endemic areas, KSHV transmission may be related to sexual activity in men, especially in male homosexuals. Higher education level and economic status are protective factors for KSHV infection.

\section{Introduction}

Kaposi's sarcoma-associated herpes virus (KSHV) also referred as human herpesvirus-8 (HHV-8), is the etiologic agent for the occurrence of Kaposi's sarcoma (KS), Primary effusion lymphoma (PEL) and Multicentric Castleman's disease (MCD) ${ }^{[1-3]}$. Since its initial discovery ${ }^{[4]}$, the molecular biology, epidemiology and pathological characteristics of this virus have been extensively studied. KSHV infection is not ubiquitous with seroprevalence, risk factors and transmission routes exhibiting considerable variations across populations in different geographic regions ${ }^{[5,6]}$. Currently, it is generally believed that sexual transmission is the main route of KSHV infection among non-endemic countries. However, there are conflicting findings about specific sexual behaviors that might be responsible for the transmission of the virus and no consensus about modes of acquisition [7]. In the North America and Europe, these non-endemic areas, KSHV infection is commonly found among HIV positive individuals, especially in the men who have sex with men (MSM) ${ }^{[8]}$, and the relationship with the number of recent male sex partners is consistent with transmission through intimate contact between men $[9,10]$.

Herpes simplex virus type 2 (HSV-2), the most common sexually transmitted agent ${ }^{[11]}$, is also an opportunistic infection in HIV/AIDS patients. Both KSHV and HSV-2 are herpesviruses establishing life-long infections in humans ${ }^{[12]}$, and acute infection, frequent reactivations from a previous infection or symptomatic episodes in the immune suppressed individuals can be devastating. The fact that the two herpesviruses are common infectious agents among $\mathrm{MSM}^{[13]}$, suggesting they may share similar transmission routes. Previous researches also had shown that demographic variables (age, gender, race), behavioral risk factors (sexual behavior, intravenous drug use), and a history diseases may affect the risk of KSHV infection and associated disease progression ${ }^{[14,15]}$, but conclusions are inconclusive. Therefore, further data to compare the epidemiologic feature of these two herpesviruses in a same group would be valuable.

In the present study, we used data from the National Health and Nutrition Examination Survey III (NHANES III), a large, population-based, cross-sectional study to explore the epidemiology of KSHV and HSV-2 in the US general adult population. Through comparing the epidemiologic characteristics of these two viruses, we aimed to advance the understanding of the potential risk factors and transmission routes of KSHV, especially sexual transmission, using a nationally representative sample. 


\section{Materials And Methods \\ Data collection}

The characteristics and seroprevalence of KSHV and HSV-2 were collected from the NHANES III

(https://wwwn.cdc.gov/nchs/nhanes/nhanes3/Default.aspx), which was a complex, multistage probability designed cross-sectional survey conducted during 1988-1994 by the National Center for Health Statistics, US Centers for Disease Control and Prevention. Data were obtained through household interviews, standardized physical examinations, and collection of biological samples at special mobile examination centers. These surveys included 39,695 individuals randomly sampled through a complex, multistage probability design ${ }^{[16]}$.

Of all NHANES III participants, 33994 were interviewed. We included a total of 18080 individuals aged 18 to 80 years. Individuals without examination were excluded $(n=1579)$. KSHV antibody was tested on 13179 participants with available remaining serum samples, and HSV-2 antibody was tested of stored sera specimens from 10720 participants.

\section{Laboratory methods}

The serum samples were stored at the NHANES surplus biorepository at $-80^{\circ} \mathrm{C}$. KSHV antibody testing was performed using ELISA to detect antibodies to the KSHV K8.1 structural glycoprotein (expressed during lytic viral replication) and open reading frame (ORF) 73 (also known as latency associated nuclear antigen, a viral regulatory protein expressed during latent infection). ELISA methods have been detailed elsewhere ${ }^{[17]}$. Both K8.1 and ORF73 were tested because some infected individuals, including those with KS, make antibodies only to lytic or latent antigens ${ }^{[18]}$. Sera were tested for HSV2 antibodies using purified glycoproteins specific for gG-2 as antigens to detect type-specific antibodies with solid-phase enzymatic immunodot assays ${ }^{[19]}$. The sensitivity of the immunodot test for recurrent, culture-proved genital HSV-2 infection is over $98 \%$, and the specificity is over $99 \%{ }^{[20]}$.

\section{Statistical analysis}

KSHV and HSV-2 seroprevalence were examined in specific subgroups of NHANES III subjects. Subjects with missing data were excluded. The participants were chosen to be 18-80 years old due to the large bias of Mexican Americans over 80 years old. Race/ethnicity was defined as non-Hispanic white, non-Hispanic black, Mexican American and others. Persons not fitting in the previous three categories were included in the others. Household size represents the total number of persons living in a single dwelling unit, both related and unrelated. Self-reported history of genital herpes was also evaluated through interview data. And participants were asked "Has a doctor or other healthcare professional ever told you that you had genital herpes?" The sexual history was asked among all participants 17-59 years old through a face-to-face interview. The seroprevalence according to whether or not have sexual behaviors, the time of first sexual intercourse, lifetime and the past year number of sex partners, and MSM activity was examined. MSM activity was presented if a man reported at least 1 male sex partner ${ }^{[17]}$.

Descriptive statistical analysis and measures of association were performed using the R programme (version 3.5.1, R core team, Vienna, Austria). Categorical variables were described as numbers or proportions. Differences for ordinal categorical variables were tested using Cochran Armitage tests. Odds ratios (OR) and 95\% confidence intervals (Cl) for all potential risk factors of KSHV and HSV-2 seropositivity among the subjects were calculated by univariate logistic regression models. Then we acquired adjusted odds ratios (aORs) after adjustment for age groups, sex and race/ ethnicity. A two-sided p-value of $<0.05$ was used as a measure of significance of the associations.

\section{Results}


The 16501 participants were predominately middle age (61.56\% under 50 years), non-Hispanic white (38.29\%) and female (53.25\%). Majority (69.09\%) of them had received middle school education and with median income (44.08\%). Most (60.42\%) were married or living as married and $59.12 \%$ were with employment.

\section{Characteristics and their associations with KSHV and HSV-2 infection}

Of the 13179 participants with available KSHV remaining serum samples, 270 individuals who were K8.1 seropositive, yielding a seroprevalence estimate of $2.05 \%$. When detecting the antibodies to the ORF73, 211 (1.60\%) were KSHV seropositive. As shown in Table 1, a significant difference for K8.1 seroprevalence was detected across age group (chisqaure $\left._{\text {trend }}=-6.71, P<0.001\right)$, infection rates increased from $1.23 \%$ among $18-29$ years old to $3.43 \%$ among $60-80$ years old (Fig. 1). K8.1 seroprevalence was higher among Mexican Americans (2.51\%) and non-Hispanic blacks (2.14\%) than among non-Hispanic whites $(1.67 \%)$. Individuals with higher level of education (chi-sqaure trend $=4.35, P<0.001)$ and economic status (chi-sqaure trend $=2.97, P=0.001$ ) had lower K8.1 seroprevalence. Compared to non-smoking participants, smokers had a higher infection rate, regardless of whether they had quitted. We observed no statistically significant associations with occupation and marital status after adjusting the age, sex and race. Patterns for ORF73 seroprevalence were similar to those for K8.1 seroprevalence, but differences across subgroups were less distinct and did not reach statistical significance (data not shown). 
Table 1

Socio-demographic and their associations with KSHV infection and HSV-2 infection in study participants

\begin{tabular}{|c|c|c|c|c|c|c|c|}
\hline \multirow[t]{2}{*}{ Characteristic } & \multirow{2}{*}{$\begin{array}{l}\text { All subjects, } \\
\text { No (\%). }\end{array}$} & \multicolumn{3}{|l|}{ KSHV } & \multicolumn{3}{|l|}{ HSV-2 } \\
\hline & & $\begin{array}{l}\text { Infection, } \\
\text { n/N (\%) }\end{array}$ & $\begin{array}{l}\text { OR } \\
(95 \% \mathrm{Cl})\end{array}$ & $\begin{array}{l}\text { aORs }^{\$} \\
(95 \% \mathrm{Cl})\end{array}$ & $\begin{array}{l}\text { Infection, } \\
\text { n/N (\%) }\end{array}$ & $\begin{array}{l}\text { OR } \\
(95 \% \mathrm{Cl})\end{array}$ & $\begin{array}{l}\text { aORs } \$ \\
(95 \% \mathrm{Cl})\end{array}$ \\
\hline All subjects & 16501 & 2.05 & & & 31.03 & & \\
\hline \multicolumn{8}{|l|}{ Gender } \\
\hline Male & 7714(46.75) & 2.13 & 1.00 & 1.00 & 24.90 & 1.00 & 1.00 \\
\hline Female & $8787(53.25)$ & 1.98 & $\begin{array}{l}0.93 \\
(0.73,1.18)\end{array}$ & $\begin{array}{l}0.95 \\
(0.75,1.21)\end{array}$ & 36.96 & $\begin{array}{l}1.77^{*} \\
(1.63,1.92)\end{array}$ & $\begin{array}{l}1.84^{\star} \\
(1.68,2.01)\end{array}$ \\
\hline$P$ & & & 0.555 & 0.613 & & $<0.001^{\star}$ & $<0.001^{\star}$ \\
\hline \multicolumn{8}{|l|}{ Age } \\
\hline $18-29$ years & $4238(25.68)$ & 1.23 & 1.00 & 1.00 & 19.53 & 1.00 & 1.00 \\
\hline $30-39$ years & 3333(20.20) & 1.44 & $\begin{array}{l}1.18 \\
(0.76,1.82)\end{array}$ & $\begin{array}{l}1.23 \\
(0.79,1.90)\end{array}$ & 35.45 & $\begin{array}{l}2.26^{\star} \\
(2.01,2.55)\end{array}$ & $\begin{array}{l}2.48^{\star} \\
(2.19,2.81)\end{array}$ \\
\hline $40-49$ years & $2588(15.68)$ & 1.92 & $\begin{array}{l}1.58^{\star} \\
(1.02,2.43)\end{array}$ & $\begin{array}{l}1.67^{*} \\
(1.08,2.57)\end{array}$ & 36.90 & $\begin{array}{l}2.41^{\star} \\
(2.13,2.73)\end{array}$ & $\begin{array}{l}2.92^{\star} \\
(2.56,3.33)\end{array}$ \\
\hline $50-59$ years & 1869(11.33) & 2.27 & $\begin{array}{l}1.87^{\star} \\
(1.17,2.95)\end{array}$ & $\begin{array}{l}2.16^{\star} \\
(1.35,3.43)\end{array}$ & 33.90 & $\begin{array}{l}2.11^{\star} \\
(1.79,2.49)\end{array}$ & $\begin{array}{l}3.07^{\star} \\
(2.57,3.66)\end{array}$ \\
\hline $60-80$ years & $4473(27.11)$ & 3.43 & $\begin{array}{l}2.86^{\star} \\
(2.02,4.14)\end{array}$ & $\begin{array}{l}3.37^{\star} \\
(2.36,4.90)\end{array}$ & 37.24 & $\begin{array}{l}2.45^{\star} \\
(2.16,2.77)\end{array}$ & $\begin{array}{l}3.81 \text { * } \\
(3.32,4.38)\end{array}$ \\
\hline$P$ & & & $<0.001 *$ & $<0.001^{*}$ & & $<0.001 *$ & $<0.001^{\star}$ \\
\hline Race/Ethnicity & & & & & & & \\
\hline Non-Hispanic white & 6318(38.29) & 1.67 & 1.00 & 1.00 & 18.84 & 1.00 & 1.00 \\
\hline Non-Hispanic black & $4840(29.33)$ & 2.14 & $\begin{array}{l}1.29 \\
(0.95,1.75)\end{array}$ & $\begin{array}{l}1.59 * \\
(1.17,2.17)\end{array}$ & 50.09 & $\begin{array}{l}4.32^{\star} \\
(3.89,4.81)\end{array}$ & $\begin{array}{l}5.52^{\star} \\
(4.92,6.19)\end{array}$ \\
\hline Mexican-American & $4664(28.26)$ & 2.51 & $\begin{array}{l}1.52^{\star} \\
(1.13,2.05)\end{array}$ & $\begin{array}{l}1.96^{\star} \\
(1.44,2.66)\end{array}$ & 26.84 & $\begin{array}{l}1.58^{\star} \\
(1.41,1.77)\end{array}$ & $\begin{array}{l}2.00 * \\
(1.78,2.25)\end{array}$ \\
\hline Other & $679(4.11)$ & 2.00 & $\begin{array}{l}1.20 \\
(0.62,2.13)\end{array}$ & $\begin{array}{l}1.42 \\
(0.73,2.52)\end{array}$ & 29.11 & $\begin{array}{l}1.77^{\star} \\
(1.40,2.23)\end{array}$ & $\begin{array}{l}2.16^{\star} \\
(1.69,2.74)\end{array}$ \\
\hline
\end{tabular}

KSHV, Kaposi's sarcoma-associated herpesvirus, the KSHV seroprevalence was used antibodies to K8.1 ;

OR, Odds ratio ; aORs, adjusted odds ratios; $\mathrm{Cl}$, confidence interval.

*Statistically significant association.

\$OR and 95\% Cl adjusted for age, sex and race/ethnicity. 


\begin{tabular}{|c|c|c|c|c|c|c|c|}
\hline \multirow[t]{3}{*}{ Characteristic } & \multirow{3}{*}{$\begin{array}{l}\text { All subjects, } \\
\text { No (\%). }\end{array}$} & \multicolumn{3}{|l|}{ KSHV } & \multicolumn{3}{|l|}{ HSV-2 } \\
\hline & & Infection, & & aORs $\$$ & Infection, & OR & aORs $\$$ \\
\hline & & & $(95 \% \mathrm{Cl})$ & $(95 \% \mathrm{Cl})$ & & $(95 \% \mathrm{Cl})$ & $(95 \% \mathrm{Cl})$ \\
\hline$P$ & & & 0.017 * & $<0.001^{\star}$ & & $<0.001^{\star}$ & $<0.001 *$ \\
\hline \multicolumn{8}{|l|}{ Household Size } \\
\hline $1-4$ & $12230(74.12)$ & 2.10 & 1.00 & 1.00 & 31.18 & 1.00 & 1.00 \\
\hline \multirow[t]{2}{*}{$5-9$} & $3875(23.48)$ & 1.88 & 0.90 & 0.99 & 30.69 & 0.98 & 0.96 \\
\hline & & & $(0.66,1.19)$ & $(0.72,1.35)$ & & $(0.89,1.07)$ & $(0.86,1.06)$ \\
\hline \multirow[t]{2}{*}{$\geq 10$} & $396(2.40)$ & 2.24 & 1.07 & 1.15 & 30.14 & 0.95 & 1.03 \\
\hline & & & $(0.45,2.13)$ & $(0.48,2.34)$ & & $(0.73,1.23)$ & $(0.77,1.36)$ \\
\hline$P$ & & & 0.619 & 0.741 & & 0.562 & 0.771 \\
\hline \multicolumn{8}{|l|}{ Education } \\
\hline 0 years & $424(2.59)$ & 6.80 & 1.00 & 1.00 & 39.46 & 1.00 & 1.00 \\
\hline \multirow[t]{2}{*}{$1-12$ years } & 11328(69.09) & 2.11 & $0.30 *$ & $0.46^{*}$ & 33.03 & 0.76 & 0.84 \\
\hline & & & $(0.19,0.49)$ & $(0.28,0.79)$ & & $(0.58,1.00)$ & $(0.63,1.13)$ \\
\hline \multirow[t]{2}{*}{$\geq 13$ years } & 4645(28.33) & 1.50 & $0.21^{\star}$ & $0.39 *$ & 25.73 & $0.53^{*}$ & $0.64 *$ \\
\hline & & & $(0.13,0.36)$ & $(0.22,0.70)$ & & $(0.40,0.71)$ & $(0.47,0.87)$ \\
\hline$P$ & & & $<0.001^{\star}$ & $0.005^{\star}$ & & $<0.001^{\star}$ & $<0.001^{\star}$ \\
\hline \multicolumn{8}{|c|}{ Poverty Income Ratio } \\
\hline$\leq 1.3$ & 4987(33.36) & 2.57 & 1.00 & 1.00 & 37.36 & 1.00 & 1.00 \\
\hline \multirow[t]{2}{*}{$1.3-3.5$} & 6589(44.08) & 1.85 & $0.71^{*}$ & $0.73^{\star}$ & 29.91 & $0.72^{\star}$ & $0.79 *$ \\
\hline & & & $(0.54,0.95)$ & $(0.55,0.98)$ & & $(0.65,0.79)$ & $(0.71,0.88)$ \\
\hline \multirow[t]{2}{*}{$\varangle 3.5$} & $3371(22.55)$ & 1.57 & $0.60 *$ & $0.65^{\star}$ & 23.78 & $0.52^{*}$ & $0.65^{\star}$ \\
\hline & & & $(0.42,0.86)$ & $(0.43,0.95)$ & & $(0.46,0.59)$ & $(0.57,0.75)$ \\
\hline$P$ & & & $0.003^{*}$ & $0.005^{\star}$ & & $<0.001^{*}$ & $<0.001^{*}$ \\
\hline \multicolumn{8}{|l|}{ Occupation } \\
\hline Unemployment & 6744(40.88) & 2.59 & 1.00 & 1.00 & 34.81 & 1.00 & 1.00 \\
\hline \multirow[t]{2}{*}{ Employment } & $9754(59.12)$ & 1.70 & $0.65^{\star}$ & 0.92 & 28.97 & $0.76^{\star}$ & 0.97 \\
\hline & & & $(0.51,0.83)$ & $(0.69,1.22)$ & & $(0.70,0.83)$ & $(0.87,1.07)$ \\
\hline$P$ & & & $<0.001^{*}$ & 0.358 & & $<0.001^{\star}$ & 0.278 \\
\hline \multicolumn{8}{|c|}{ KSHV, Kaposi's sarcoma-associated herpesvirus, the KSHV seroprevalence was used antibodies to K8.1; } \\
\hline \multicolumn{8}{|c|}{ OR, Odds ratio ; aORs, adjusted odds ratios; $\mathrm{Cl}$, confidence interval. } \\
\hline \multicolumn{8}{|c|}{ *Statistically significant association. } \\
\hline 2 an & ge se & race/eth & & & & & \\
\hline
\end{tabular}




\begin{tabular}{|c|c|c|c|c|c|c|c|}
\hline \multirow[t]{2}{*}{ Characteristic } & \multirow{2}{*}{$\begin{array}{l}\text { All subjects, } \\
\text { No (\%). }\end{array}$} & \multicolumn{3}{|l|}{ KSHV } & \multicolumn{3}{|l|}{ HSV-2 } \\
\hline & & $\begin{array}{l}\text { Infection, } \\
\text { n/N (\%) }\end{array}$ & $\begin{array}{l}\text { OR } \\
(95 \% \mathrm{Cl})\end{array}$ & $\begin{array}{l}\text { aORs\$ } \\
(95 \% \mathrm{Cl})\end{array}$ & $\begin{array}{l}\text { Infection, } \\
\text { n/N (\%) }\end{array}$ & $\begin{array}{l}\text { OR } \\
(95 \% \mathrm{Cl})\end{array}$ & $\begin{array}{l}\text { aORs\$ } \\
(95 \% \mathrm{Cl})\end{array}$ \\
\hline \multicolumn{8}{|l|}{ Marital Status } \\
\hline Never married & $3337(20.26)$ & 1.76 & 1.00 & 1.00 & 31.27 & 1.00 & 1.00 \\
\hline \multirow{2}{*}{$\begin{array}{l}\text { Married/Living as } \\
\text { married }\end{array}$} & $9950(60.42)$ & 1.87 & 1.07 & 0.69 & 31.20 & 1.00 & 0.96 \\
\hline & & & $(0.77,1.50)$ & $(0.47,1.02)$ & & $(0.90,1.11)$ & $(0.86,1.08)$ \\
\hline \multirow{2}{*}{$\begin{array}{l}\text { Separated/Divorced } \\
\text { / Widowed }\end{array}$} & $3182(19.32)$ & 2.94 & $1.70^{\star}$ & 0.93 & 30.32 & 0.96 & 0.93 \\
\hline & & & $(1.18,2.47)$ & $(0.61,1.45)$ & & $(0.84,1.08)$ & $(0.81,1.06)$ \\
\hline$P$ & & & $0.003^{*}$ & 0.781 & & 0.478 & 0.346 \\
\hline \multicolumn{8}{|l|}{ Smoking Status } \\
\hline No-smoking & $8209(49.75)$ & 1.73 & 1.00 & 1.00 & 27.85 & 1.00 & 1.00 \\
\hline \multirow[t]{2}{*}{ On-smoking } & $4438(26.90)$ & 1.95 & 1.13 & 1.21 & 36.12 & $1.47^{\star}$ & $1.71 *$ \\
\hline & & & $(0.83,1.52)$ & $(0.88,1.65)$ & & $(1.33,1.61)$ & $(1.53,1.90)$ \\
\hline \multirow[t]{2}{*}{ Quit-smoking } & $3853(23.35)$ & 2.86 & $1.67^{\star}$ & $1.44^{*}$ & 31.68 & $1.20 *$ & $1.41^{\star}$ \\
\hline & & & $(1.26,2.21)$ & $(1.06,1.95)$ & & $(1.08,1.34)$ & $(1.24,1.59)$ \\
\hline$P$ & & & $<0.001 *$ & $0.023^{\star}$ & & $<0.001 *$ & $<0.001 *$ \\
\hline \multicolumn{8}{|l|}{ Drinking Status } \\
\hline No-drinking & 2838(17.98) & 2.33 & 1.00 & 1.00 & 29.03 & 1.00 & 1.00 \\
\hline \multirow[t]{2}{*}{ Drinking } & 12943(82.02) & 2.03 & 0.87 & 1.07 & 31.26 & 1.11 & $1.54^{*}$ \\
\hline & & & $(0.65,1.19)$ & $(0.77,1.47)$ & & $(1.00,1.24)$ & $(1.36,1.75)$ \\
\hline$P$ & & & 0.369 & 0.602 & & 0.062 & $<0.001 *$ \\
\hline \multicolumn{8}{|c|}{ KSHV, Kaposi's sarcoma-associated herpesvirus, the KSHV seroprevalence was used antibodies to K8.1 ; } \\
\hline \multicolumn{8}{|c|}{ OR, Odds ratio ; aORs, adjusted odds ratios; $\mathrm{Cl}$, confidence interval. } \\
\hline \multicolumn{8}{|c|}{ *Statistically significant association. } \\
\hline 2 and $95 \% \mathrm{Cl}$ adiu & for age sex & sen/ott & & & & & \\
\hline
\end{tabular}

Overall, the seroprevalence of HSV-2 were $31.03 \%$ (3326 out of 10720). Of those testing positive for HSV-2 infection, $95.12 \%$ reported never been diagnosed as genital herpes. Female (36.96\%) and non-Hispanic black (50.09\%) individuals had a higher risk of HSV-2 infection. There is a strong association between HSV-2 seropositivity and age, with prevalence generally rising rapidly between the ages of 18 and 39 years before becoming stable. Compared with KSHV infection, in addition to education, economic status and smoking status, drinking was also correlated with HSV-2 seropositivity. Drinking increased the risk of HSV-2 infection after adjustment $(\mathrm{OR}=1.54 ; 95 \% \mathrm{Cl} 1.361 .75)$.

\section{Co-infections and their associations with KSHV and HSV-2 infection}


The overall co-infection rate of Hepatitis B virus (HBV, HBsAg), Hepatitis C virus (HCV), Herpes simplex virus type 1 (HSV-1), HSV-2 and Cytomegalovirus (CMV) are 7.94\%, 2.21\%, 1.99\%, 2.41\% and 2.40\%, respectively. Univariate logistic regression analyses indicated that both HSV-2 and CMV infection were significantly associated with K8.1 serepositivity. Those who were infected with HSV-2 (OR 1.58, 95\% Cl 1.09 2.27) or CMV (OR 2.12, 95\% Cl 1.37 3.43) were more likely to be infected with KSHV. Such associations were still significant after adjusting sex, age, race, education level and economic status for potential confounders (Table 2). However, there was no statistically significant association between KSHV infection and HBV, HCV and HSV-1 infection. Different with KSHV co-infection, participants infected with HCV were more likely to be infected with HSV-2 (OR 2.16, 95\% Cl 1.63 2.85). 
Table 2

Serostatus of HBsAg, HCV, HSV-1 and CMV and Co-infection by KSHV and HSV-2 in study participants

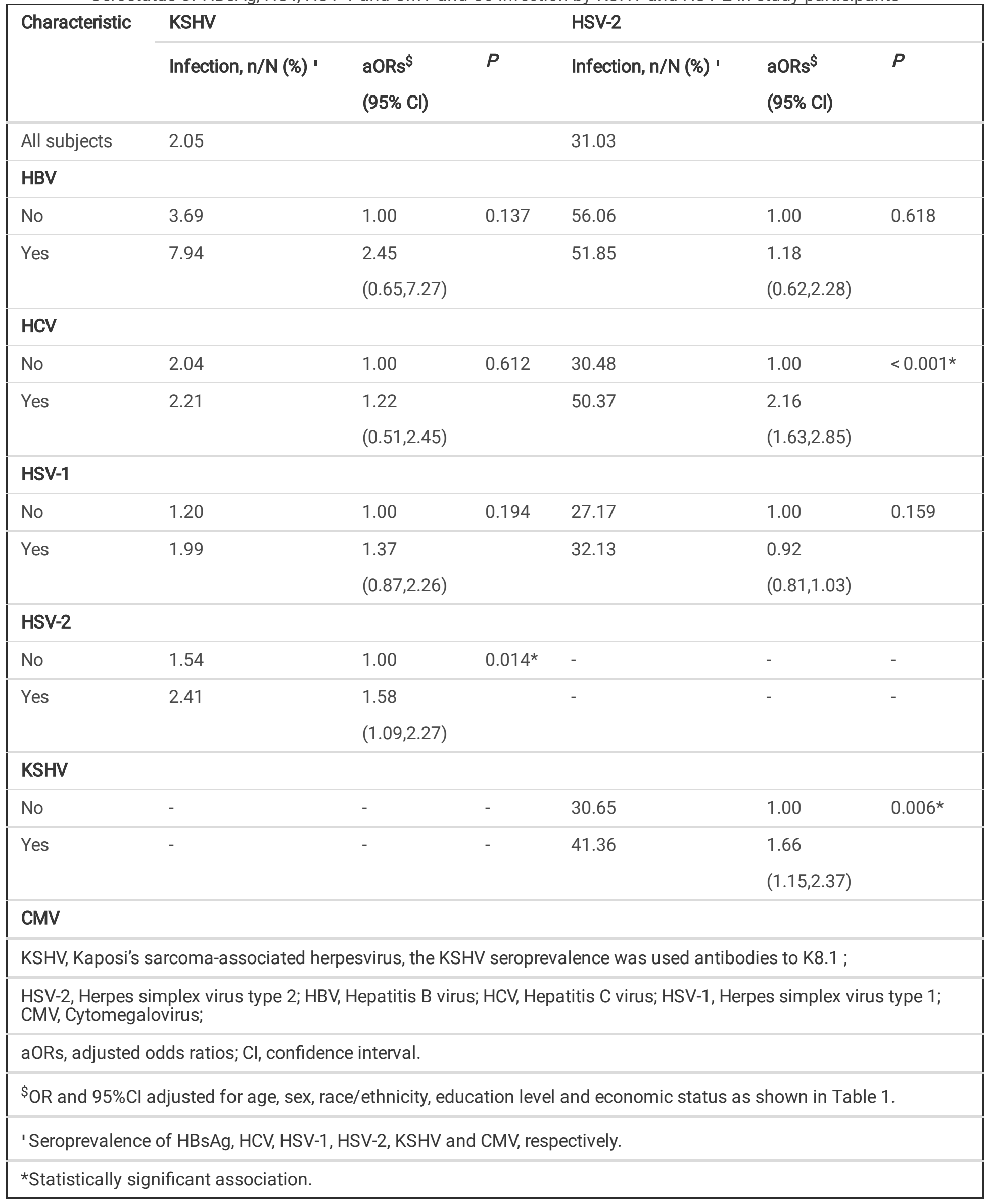




\begin{tabular}{|c|c|c|c|c|c|c|}
\hline \multirow[t]{3}{*}{ Characteristic } & \multicolumn{3}{|l|}{ KSHV } & \multicolumn{3}{|l|}{ HSV-2 } \\
\hline & \multirow[t]{2}{*}{ Infection, $n / N(\%) '$} & aORs\$ & $P$ & Infection, n/N (\%) ' & aORs & $P$ \\
\hline & & \multicolumn{3}{|c|}{$(95 \% \mathrm{Cl})$} & \multicolumn{2}{|c|}{$(95 \% \mathrm{Cl})$} \\
\hline No & 0.88 & 1.00 & $0.001 *$ & 17.43 & 1.00 & $<0.001^{*}$ \\
\hline \multirow[t]{2}{*}{ Yes } & \multirow[t]{2}{*}{2.40} & 2.12 & & \multirow[t]{2}{*}{35.55} & 1.59 & \\
\hline & & \multicolumn{2}{|c|}{$(1.37,3.43)$} & & \multicolumn{2}{|c|}{$(1.40,1.81)$} \\
\hline \multicolumn{7}{|c|}{ KSHV, Kaposi's sarcoma-associated herpesvirus, the KSHV seroprevalence was used antibodies to K8.1; } \\
\hline \multicolumn{7}{|c|}{$\begin{array}{l}\text { HSV-2, Herpes simplex virus type 2; HBV, Hepatitis B virus; HCV, Hepatitis C virus; HSV-1, Herpes simplex virus type } 1 \text {; } \\
\text { CMV, Cytomegalovirus; }\end{array}$} \\
\hline \multicolumn{7}{|c|}{ aORs, adjusted odds ratios; $\mathrm{Cl}$, confidence interval. } \\
\hline \multicolumn{7}{|c|}{ \$OR and 95\% Cl adjusted for age, sex, race/ethnicity, education level and economic status as shown in Table 1.} \\
\hline \multicolumn{7}{|c|}{ ' Seroprevalence of HBsAg, HCV, HSV-1, HSV-2, KSHV and CMV, respectively. } \\
\hline \multicolumn{7}{|c|}{ *Statistically significant association. } \\
\hline
\end{tabular}

\section{Sexual behaviors and their associations with KSHV and HSV-2 infection}

All sexual behaviors included were strongly associated with HSV-2 infection, confirmed HSV-2 was a common sexually transmitted infection (Table 3). In contrast to HSV-2, the prevalence of KSHV did not follow the pattern expected of a sexually transmitted infection. After adjusting the age, the duration of sexual activity did not show significant association with KSHV seropositivity. Only participants with homosexual activity showed an elevated risk of KSHV infection. Of all subjects, 83 reported a history of homosexual activity (weighted prevalence, 1.07\% among all male participants) and 5 out of 83 were K8.1 positive. Participants with MSM activity increased the risk of KSHV infection with fourfold $(\mathrm{OR}=4.98$; $95 \% \mathrm{Cl} 1.69$ 11.77; $\mathrm{P}<0.001)$. 
Sexual behaviors and their associations with KSHV infection and HSV-2 infection in study participants

\begin{tabular}{|c|c|c|c|c|c|c|c|}
\hline \multirow[t]{2}{*}{ Characteristic } & \multirow{2}{*}{$\begin{array}{l}\text { All subjects, } \\
\text { No (\%). }\end{array}$} & \multicolumn{3}{|l|}{ KSHV } & \multicolumn{3}{|l|}{ HSV-2 } \\
\hline & & $\begin{array}{l}\text { Infection, } \\
\text { n/N (\%) }\end{array}$ & OR $(95 \% \mathrm{Cl})$ & $\begin{array}{l}\text { aORs\$ }(95 \% \\
\mathrm{Cl})\end{array}$ & $\begin{array}{l}\text { Infection, } \\
\text { n/N (\%) }\end{array}$ & OR (95\% Cl) & $\begin{array}{l}\text { aORsF } \\
(95 \% \mathrm{Cl})\end{array}$ \\
\hline \multicolumn{8}{|l|}{ Sexual } \\
\hline No sex & $344(2.98)$ & 1.82 & 1.00 & 1.00 & 5.93 & 1.00 & 1.00 \\
\hline \multirow[t]{2}{*}{ sex } & 11185(97.20) & 1.62 & 0.89 & 0.72 & 30.27 & $6.89 *$ & $4.64^{\star}$ \\
\hline & & & $(0.40,2.52)$ & $(0.31,2.06)$ & & $(4.23,12.15)$ & $(2.82,8.26)$ \\
\hline$P$ & & & 0.796 & 0.532 & & $<0.001 *$ & $<0.001^{\star}$ \\
\hline \multicolumn{8}{|c|}{ Initial sexual Age } \\
\hline$\leq 18$ years & $8339(74.56)$ & 1.74 & 1.00 & 1.00 & 32.39 & 1.00 & 1.00 \\
\hline \multirow[t]{2}{*}{$19-29$} & $2776(24.82)$ & 1.28 & 0.73 & 0.72 & 23.71 & $0.65^{\star}$ & $0.56^{\star}$ \\
\hline & & & $(0.48,1.08)$ & $(0.47,1.08)$ & & $(0.58,0.73)$ & $(0.49,0.64)$ \\
\hline \multirow[t]{2}{*}{$\geq 30$} & $70(0.63)$ & 1.72 & 0.99 & 0.81 & 25.00 & 0.70 & $0.47 *$ \\
\hline & & & $(0.06,4.56)$ & $(0.05,3.85)$ & & $(0.34,1.37)$ & $(0.22,0.93)$ \\
\hline$P$ & & & 0.154 & 0.083 & & $<0.001 *$ & $<0.001 *$ \\
\hline \multicolumn{8}{|l|}{ Sex Partners } \\
\hline 1 & 2418(21.91) & 1.40 & 1.00 & 1.00 & 16.40 & 1.00 & 1.00 \\
\hline \multirow[t]{2}{*}{$2-4$} & $3481(31.54)$ & 1.61 & 1.15 & 1.13 & 30.26 & $2.21^{\star}$ & $2.38^{\star}$ \\
\hline & & & $(0.72,1.86)$ & $(0.70,1.84)$ & & $(1.90,2.58)$ & $(2.02,2.80)$ \\
\hline \multirow[t]{2}{*}{$5-9$} & $2249(20.38)$ & 1.81 & 1.29 & 1.23 & 35.60 & $2.82^{\star}$ & $3.48^{\star}$ \\
\hline & & & $(0.78,2.15)$ & $(0.72,2.09)$ & & $(2.40,3.32)$ & $(2.91,4.18)$ \\
\hline \multirow[t]{2}{*}{$\geq 10$} & 2890(26.18) & 1.66 & 1.18 & 1.06 & 35.90 & $2.85^{\star}$ & $4.85^{\star}$ \\
\hline & & & $(0.73,1.95)$ & $(0.61,1.84)$ & & $(2.45,3.34)$ & $(4.03,5.85)$ \\
\hline$P$ & & & 0.463 & 0.574 & & $<0.001 *$ & $<0.001 *$ \\
\hline \multicolumn{8}{|c|}{ The duration of sexual activity } \\
\hline $0-20$ & $6243(54.15)$ & 1.25 & 1.00 & 1.00 & 24.14 & 1.00 & 1.00 \\
\hline \multirow[t]{2}{*}{$21-30$} & $2946(25.55)$ & 2.07 & $1.67^{\star}$ & 1.53 & 36.91 & $1.84^{\star}$ & $1.47 *$ \\
\hline & & & $(1.15,2.40)$ & $(0.90,2.58)$ & & $(1.65,2.05)$ & $(1.25,1.72)$ \\
\hline
\end{tabular}

KSHV, Kaposi's sarcoma-associated herpesvirus, the KSHV seroprevalence was used antibodies to K8.1 ; OR, Odds ratio ; aORs, adjusted odds ratios; $\mathrm{Cl}$, confidence interval.

*Statistically significant association.

\$OR and 95\% Cl adjusted for age, sex and race/ethnicity as shown in Table 1. 


\begin{tabular}{|c|c|c|c|c|c|c|c|}
\hline \multirow[t]{2}{*}{ Characteristic } & \multirow{2}{*}{$\begin{array}{l}\text { All subjects, } \\
\text { No (\%). }\end{array}$} & \multicolumn{3}{|l|}{ KSHV } & \multicolumn{3}{|l|}{ HSV-2 } \\
\hline & & $\begin{array}{l}\text { Infection, } \\
\text { n/N (\%) }\end{array}$ & OR $(95 \% \mathrm{Cl})$ & $\begin{array}{l}\text { aORs\$ }(95 \% \\
\mathrm{Cl})\end{array}$ & $\begin{array}{l}\text { Infection, } \\
\text { n/N (\%) }\end{array}$ & OR $(95 \% \mathrm{Cl})$ & $\begin{array}{l}\text { aORsF } \\
(95 \% \mathrm{Cl})\end{array}$ \\
\hline \multirow[t]{2}{*}{$31-40$} & 1812(15.72) & 1.95 & $1.57^{*}$ & 1.29 & 35.90 & $1.76^{*}$ & $1.83^{*}$ \\
\hline & & & $(1.00,2.42)$ & $(0.58,2.81)$ & & $(1.53,2.03)$ & $(1.43,2.34)$ \\
\hline \multirow[t]{2}{*}{$\geq 40$} & $528(4.58)$ & 2.57 & $2.08 *$ & 1.58 & 40.53 & $2.14^{\star}$ & $2.46^{*}$ \\
\hline & & & $(1.00,3.89)$ & $(0.54,4.36)$ & & $(1.66,2.76)$ & $(1.69,3.59)$ \\
\hline$P$ & & & $0.004^{*}$ & 0.320 & & $<0.001 *$ & $0.002^{\star}$ \\
\hline \multicolumn{8}{|c|}{ Sex Partners in the past year } \\
\hline 0 & 1110(9.92) & 2.62 & 1.00 & 1.00 & 32.30 & 1.00 & 1.00 \\
\hline \multirow[t]{2}{*}{1} & 8188(73.20) & 1.52 & $0.57 *$ & $0.62^{\star}$ & 28.83 & 0.85 & 1.01 \\
\hline & & & $(0.37,0.92)$ & $(0.40,1.01)$ & & $(0.72,1.00)$ & $(0.84,1.21)$ \\
\hline \multirow[t]{2}{*}{$2-4$} & 1588(14.20) & 1.37 & $0.52^{*}$ & 0.54 & 35.17 & 1.14 & $1.55^{\star}$ \\
\hline & & & $(0.28,0.95)$ & $(0.28,1.01)$ & & $(0.94,1.38)$ & $(1.25,1.94)$ \\
\hline \multirow[t]{2}{*}{$5-9$} & 219(1.96) & 2.84 & 1.09 & 1.08 & 33.33 & 1.05 & $1.78^{\star}$ \\
\hline & & & $(0.36,2.67)$ & $(0.35,2.77)$ & & $(0.73,1.49)$ & $(1.20,2.60)$ \\
\hline \multirow[t]{2}{*}{$\geq 10$} & $81(0.72)$ & - & - & - & 41.27 & 1.47 & $2.07 *$ \\
\hline & & & & & & $(0.86,2.48)$ & $(1.17,3.64)$ \\
\hline$P$ & & & 0.132 & 0.205 & & $0.002^{*}$ & $<0.001^{*}$ \\
\hline \multicolumn{8}{|c|}{ Sexual preference } \\
\hline No-MSM & 4922(98.34) & 1.63 & 1.00 & 1.00 & 22.30 & 1.00 & 1.00 \\
\hline \multirow[t]{2}{*}{ MSM } & $83(1.66)$ & 7.25 & $4.71^{\star}$ & $4.98^{\star}$ & 39.68 & $2.29 *$ & $2.85^{\star}$ \\
\hline & & & $(1.61,11.03)$ & $(1.69,11.77)$ & & $(1.36,3.80)$ & $(1.65,4.87)$ \\
\hline$P$ & & & $0.001^{*}$ & $<0.001^{\star}$ & & $0.001 *$ & $<0.001^{*}$ \\
\hline \multicolumn{8}{|c|}{ KSHV, Kaposi's sarcoma-associated herpesvirus, the KSHV seroprevalence was used antibodies to K8.1 ; } \\
\hline \multicolumn{8}{|c|}{ OR, Odds ratio ; aORs, adjusted odds ratios; $\mathrm{Cl}$, confidence interval. } \\
\hline \multicolumn{8}{|c|}{ *Statistically significant association. } \\
\hline
\end{tabular}

\section{Discussion}

Despite consistent increasing understanding of KSHV biology and its clinical manifestations, progress has not been significantly made in its epidemiology which in turn hampered the management of KSHV-associated diseases and public health. In this study, we based on a large general population to explore the epidemiologic feature of KSHV infection. The 
seropositivity of KSHV was $2.05 \%$, higher than previous study ${ }^{[17]}$. And the results supported that KSHV appears to be sexually transmitted in MSM in this low-risk, heterosexual population.

In the present study, we explored the potential correlates of sexual behaviors with KSHV and HSV-2 infection, and found that KSHV transmission may be related to sexual activity in men, especially in male homosexuals. Our results showed MSM had an approximately fivefold increased risk of KSHV infection $(\mathrm{OR}=4.98)$, which was generally consistent with previous studies ${ }^{[21-23]}$. Thus, the transmission of KSHV in MSM is clearly associated with sexual risk factors, whereas evidence of heterosexual transmission of KSHV is less consistent. Homosexual individuals were more likely to have oral sex and with multiple sexual partners ${ }^{[24]}$. While KSHV can be detected common in the saliva of infected subjects, indicating that saliva is the most likely source of KSHV during transmission between MSM ${ }^{[25]}$. Our results did not show a clear between the number of sexual partners and KSHV seropositivity $(P=0.574)$. This may be affected by the methods of data acquisition. The data on sexual behaviors are obtained through interview with gerneral population, which may cause some selection biases.

As expected, there was an increased HSV-2 prevalence with earlier initial sexual age and number of sexual partners in life. Meanwhile, HSV-2 infection risk increased about threefold (OR = 2.85) among MSM. High prevalence of the two viral among MSM suggests that the two viral may facilitate the acquisition of each other through sexual contacts with this population and further supported KSHV transmission may be related to male homosexuals. HSV-2 infection can contribute to the sexual transmission of KSHV and may also be associated with reactivation of KSHV latent infection. Previous studies in Northern Cameroon showed similar results ${ }^{[7]}$. HSV-2 infection is known to recruit at the site of replication white blood cells including B and CD8 lymphocyte that can be infected by KSHV. In addition, reactivation of HSV-2 might increase the load at mucosal and systemic levels of other viruses including KSHV, HIV [26], and HCV [27]. Viral shedding and transmission to sex partners can occur in the absence of symptoms or a noticeable lesion ${ }^{[28]}$. Noticeably, our study revealed that $95.12 \%$ of HSV-2 infections were asymptomatic or unrecognized, and this could exacerbate the transmission of KSHV. We also found that HSV-2 positive subjects were more likely to be infected with KSHV (OR $=1.58)$, this association implies that HSV-2 and KSHV may share similar transmission routes.

Furthermore, we have also confirmed KSHV infection was associated with increasing age, compared with subjects between 18-29 years old, the risk among 50-59 years old was increased about twofold $(\mathrm{OR}=2.27)$ and among $60-80$ years old was increased about threefold $(\mathrm{OR}=3.43)$. Unlike the relatively stable age pattern of HSV-2 seroprevalence, the prevalence of KSHV increases steadily with age, suggesting that KSHV may be not sexually transmitted. The role of education level and economic status against KSHV have been explored by other investigators, however, the results are controversial [29]. Our study suggests that higher education level and higher economic status are protective factors for KSHV infection.

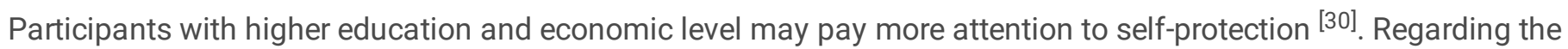
household size, we did not found correlation with KSHV seroprevalence, however, we did not know people who living together whether sharing public utensils. Thus, we cannot infer the possible transmission route through saliva from the household size.

Defining modes of transmission of KSHV remains a challenge, especially in non-endemic areas. In this study, we were able to compare the well-known pattern of sexually related risk factors for HSV-2, with that seen for KSHV in a community at risk of both. The implication of our findings is that KSHV appears to be sexually transmitted in MSM, although transmission among heterosexuals is less likely.

Some limitation should be noted here. First, there is a lack of standard KSHV serological assay. We are based on the results from the EIAs, which may different with other methods. Second, NHANES III lacks data on specific sexual practices are unavailable, which prevented us from exploring potential routes of sexual transmission in more details. 


\section{Conclusion}

In non-endemic areas, KSHV transmission may be related to sexual activity in men, especially in male homosexuals. Our results clarify the transmission routes of KSHV infection, which would ultimately help the development of interventions that prevent infection.

\section{Abbreviations}

KSHV: Kaposi's sarcoma-associated herpes virus; HHV-8: Human herpesvirus-8; KS: Kaposi's sarcoma; PEL: Primary effusion lymphoma; MCD: Multicentric Castleman's disease; MSM: Men who have sex with men; HSV-2: Herpes simplex virus type 2; NHANES III: National Health and Nutrition Examination Survey III ; ORF: Open reading frame.

\section{Declarations}

\section{Ethics approval and consent to participate}

Not applicable.

\section{Consent for publication}

Not applicable.

\section{Availability of data and materials}

The datasets analysed during the current study were collected from the NHANES III (https://wwwn.cdc.gov/nchs/nhanes/nhanes3/Default.aspx), which was a complex, multistage probability designed cross-sectional survey conducted during 1988-1994 by the National Center for Health Statistics, US Centers for Disease Control and Prevention.

\section{Competing interests}

The authors declare that they have no competing interests.

\section{Funding}

This work was supported by National Natural Science Foundation of China (81772170) and by Special project on national science and technology basic resources investigation (2019FY101103).

\section{Authors' contributions}

All authors contributed to the study conception and design. $X Z, Y Y X$ were responsible for data collection. $X Z$, $Y L$ were responsible for sorting and cleaning the data. Data analysis was performed by ZQL and HBY. XZ is responsible for 
interpreting the results and drafting the article. TJZ is responsible for revising it critically for important intellectual content and final approval.

\section{Acknowledgements}

We appreciate the work by National Health and Nutrition Examination Survey III collaborators. And we are grateful to all the participants for their cooperation to make this study possible.

\section{Disclaimer}

The funders did not play a role in the design, conduct or analysis of the study, nor in the drafting of this manuscript.

\section{References}

1. Whitby $D$, et al. Detection of Kaposi sarcoma associated herpesvirus in peripheral blood of HIV-infected individuals and progression to Kaposi's sarcoma. Lancet. 1995;346(8978):799-802.

2. Cesarman E, et al. Kaposi's sarcoma-associated herpesvirus-like DNA sequences in AIDS-related body-cavity-based lymphomas. N Engl J Med. 1995;332(18):1186-91.

3. Soulier J, et al. Kaposi's sarcoma-associated herpesvirus-like DNA sequences in multicentric Castleman's disease. Blood. 1995;86(4):1276-80.

4. Chang Y, et al. Identification of herpesvirus-like DNA sequences in AIDS-associated Kaposi's sarcoma. Science. 1994;266(5192):1865-9.

5. Pfeiffer RM, et al. Geographic heterogeneity of prevalence of the human herpesvirus 8 in sub-Saharan Africa: clues about etiology. Ann Epidemiol. 2010;20(12):958-63.

6. de Sanjose S, et al. Geographic Variation in the Prevalence of Kaposi Sarcoma-Associated Herpesvirus and Risk Factors for Transmission. J Infect Dis. 2009;199(10):1449-56.

7. Volpi A, et al. Correlates of human herpes virus-8 and herpes simplex virus type 2 infections in Northern Cameroon. $J$ Med Virol. 2004;74(3):467-72.

8. Qu LR, Jenkins F, Triulzi DJ. Human herpesvirus 8 genomes and seroprevalence in United States blood donors. Transfusion. 2010;50(5):1050-6.

9. Qu L, Jenkins F, Triulzi DJ. Human herpesvirus 8 genomes and seroprevalence in United States blood donors. Transfusion. 2010;50(5):1050-6.

10. O'Brien TR, et al. Evidence for concurrent epidemics of human herpesvirus 8 and human immunodeficiency virus type 1 in US homosexual men: rates, risk factors, and relationship to Kaposi's sarcoma. J Infect Dis. 1999;180(4):1010-7.

11. Beydoun HA, et al. Socio-demographic and behavioral correlates of herpes simplex virus type 1 and 2 infections and co-infections among adults in the USA. International Journal of Infectious Diseases. 2010;14:E154-60.

12. Chemaitelly $\mathrm{H}$, et al., Characterizing herpes simplex virus type 1 and type 2 seroprevalence declines and epidemiological association in the United States. Plos One, 2019. 14(6).

13. Hechter RC, et al. Herpes simplex virus type 2 (HSV-2) as a coronary atherosclerosis risk factor in HIV-infected men: Multicenter AIDS Cohort Study. Atherosclerosis. 2012;223(2):433-6.

14. Fu B, et al. Seroprevalence of Kaposi's sarcoma-associated herpesvirus and risk factors in Xinjiang, China. J Med Virol. 2009;81(8):1422-31.

15. Fang Q, et al. Prevalence of Kaposi's sarcoma-associated herpesvirus among intravenous drug users: a systematic review and meta-analysis. Virol Sin. 2017;32(5):415-22. 
16. National Center for Health Statistics (NCHS). Vital health statistics: plan and operation of the third National Health and Nutrition Examination Survey, 1988-94. Hyattsville, MD: NCHS, 1994.

17. Engels EA, et al. Risk factors for human herpesvirus 8 infection among adults in the United States and evidence for sexual transmission. J Infect Dis. 2007;196(2):199-207.

18. Biggar RJ, et al. Antibody reactivity to latent and lytic antigens to human herpesvirus-8 in longitudinally followed homosexual men. J Infect Dis. 2003;187(1):12-8.

19. Fleming DT. McQuillan GM, Johnson RE, et al. Herpes simplex virus type 2 in the United States, 1976 to $1994 . N$ Engl J Med 1997; 337: 1105-11..

20. Lee FK, et al. Detection of herpes simplex virus type 2-specific antibody with glycoprotein G. J Clin Microbiol. 1985;22(4):641-4.

21. Wang J, et al. Prevalence of Kaposi's sarcoma associated herpesvirus among men attending sexually transmitted infections clinics in Anhui, China. J Med Virol. 2016;88(2):304-11.

22. Liu Z, et al. High seroprevalence of human herpesvirus 8 and herpes simplex virus 2 infections in men who have sex with men in Shanghai, China. J Med Virol. 2017;89(5):887-94.

23. Martro E, et al. Risk factors for human Herpesvirus 8 infection and AIDS-associated Kaposi's sarcoma among men who have sex with men in a European multicentre study. Int J Cancer. 2007;120(5):1129-35.

24. Minhas V, Wood C. Epidemiology and transmission of Kaposi's sarcoma-associated herpesvirus. Viruses. 2014;6(11):4178-94.

25. Malope BI, et al. No evidence of sexual transmission of Kaposi's sarcoma herpes virus in a heterosexual South African population. Aids. 2008;22(4):519-26.

26. Schacker T, et al. Frequent recovery of HIV-1 from genital herpes simplex virus lesions in HIV-1-infected men. JAMA. 1998;280(1):61-6.

27. Okafor IM, Ugwu SO, Okoroiwu HU. Hepatitis C virus infection and its associated factors among prisoners in a Nigerian prison. BMC Gastroenterol. 2020;20(1):360.

28. Seroprevalence of Herpes Simplex Virus Type 2 Among Persons Aged 14-49 Years-United States, 2005-2008 (Reprinted from MMWR, vol 59, pg 456-459, 2010). Jama-Journal of the American Medical Association, 2010. 304(8): p. 849-850.

29. Semango GP, et al., Prevalence and associated risk factors for Kaposi's sarcoma among HIV-positive patients in a referral hospital in Northern Tanzania: a retrospective hospital-based study. BMC Cancer, 2018. 18(1).

30. Wakeham K, et al. Risk factors for seropositivity to Kaposi sarcoma-associated herpesvirus among children in Uganda. J Acquir Immune Defic Syndr. 2013;63(2):228-33.

\section{Figures}




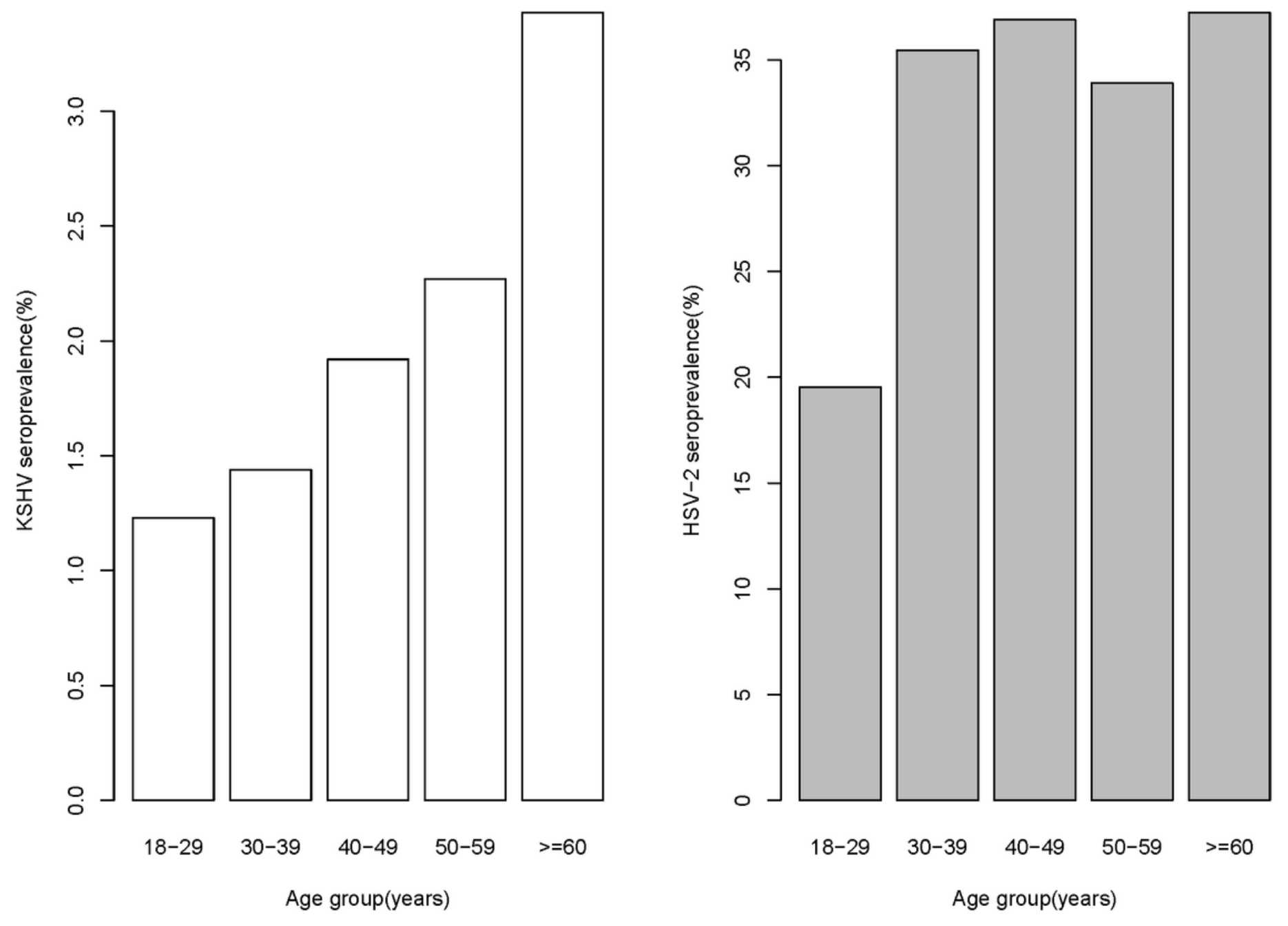

Figure 1

The age distribution of KSHV and HSV2 prevalence among participants. 